craftworks to convert and clean up original dataset before the beginning of statistical analysis. To improve procedure of data management by raising interoperability of dataset, we technically examined a standard-based object-oriented data model for a common database between data entry system and statistical analysing system, and estimated how the process of data management was changed with the new data model.

Methods We adopted the Archetypes data model, which is a standard of ISO 13606, as the storage for collected dataset and the $\mathrm{R}$ language and environment for statistical computing as the statistical software. An example dataset was sampled from a cohort study. We simulated to develop an exclusive data entry sheet for the study. To estimate effect of introducing Archetypes database, we enumerated operations which will be required to build the system, input, review, clean, transfer, and analysis the example dataset. The complexity of each operation was estimated. For control, same estimation was performed on a system with traditional database.

Results Archetypes approach was expected to require more complicated procedures to build the data entry system than traditional approach, however, more software components was expected to reusable between other datasets. Both approaches were expected to require similar number of operations to manage datasets.

Conclusion For electrical data collection of epidemiological study, introduction of standardised data model might lead to efficient development of data entry system.

\section{P1-44 APPLICATION OF A NOVEL METHOD TO ESTIMATE INCIDENCE OF HEPATITIS C AMONG INJECTING DRUG USERS IN SCOTLAND}

doi:10.1136/jech.2011.142976c.37

\begin{abstract}
${ }^{1}$ Norah Palmateer, ${ }^{*}{ }^{2}$ Elizabeth Allen, ${ }^{2}$ Avril Taylor, ${ }^{1,3}$ Sharon Hutchinson, ${ }^{1}$ David Goldberg. ${ }^{1}$ Health Protection Scotland, Glasgow, UK; ${ }^{2}$ University of the West of Scotland, Paisley, UK; ${ }^{3}$ Department of Mathematics and Statistics, University of Strathclyde, Glasgow, UK
\end{abstract}

Background Prevalence of the hepatitis C virus (HCV) among injecting drug users (IDUs) in Scotland is high. Through an HCV Action Plan, the Scottish Government has invested significantly in harm reduction interventions with the goal of reducing HCV transmission among IDUs. In evaluating the effectiveness of interventions, estimates of the incidence, rather than the prevalence, of HCV are essential. The traditional approach to measuring incidence-follow-up of a cohort of uninfected individuals to measure seroconversion-can, however, be costly and suffer high participant attrition rates. We report the first large-scale, national application of a novel method designed to determine incidence of HCV using a cross-sectional design.

Methods During 2008-2009, IDUs were recruited from needle exchange services, completed an interviewer-administered questionnaire and provided a dried blood spot for anonymous HCVantibody (anti-HCV) and HCV-RNA testing. Incident infections were defined as individuals who were anti-HCV negative and HCVRNA positive.

Results Prevalence of anti-HCV was 55\% among the 2563 participants. Among anti-HCV negative respondents, twenty-one were HCV-RNA positive, yielding an incidence estimate of 12.1 per 100 person-years (corresponding to a viraemic pre-seroconversion window period of 51 days). Individuals currently receiving methadone had lower odds of incident infection (adjusted OR 0.15; 95\% CI 0.035 to 0.68 ) relative to those on methadone in the previous 6 months (but not currently).
Conclusions This approach to measuring incidence will allow us to gauge the impact of preventive interventions associated with the HCV Action Plan.

\section{P1-45 NOVEL APPLICATION OF LOT QUALITY ASSURANCE SAMPLING: AS A QUALITY CONTROL TOOL IN CENSUS VERIFICATION}

doi:10.1136/jech.2011.142976c.38

${ }^{1} \mathrm{~B}$ K Patro, ${ }^{2} \mathrm{~S}$ Saha, ${ }^{2} \mathrm{~K}$ Anand, ${ }^{2} \mathrm{~S} \mathrm{~K}$ Kapoor. ${ }^{1} \mathrm{P}$ GIMER school of public health Chandigarh, India; ${ }^{2}$ Centre for community medicine, AIIMS, New Delhi, India

Introduction Lot Quality Assurance Sampling (LOAS) was developed as technique for quality assurance in industrial production. The use of LOAS in health sciences is limited to coverage evaluations of different health activities such as immunisation coverage, antenatal care coverage, elimination of neonatal tetanus and coverage iodised salt and others. LOAS has been accepted as rapid epidemiological survey method. The present study was carried out to explore the feasibility of using LOAS in Census Verification.

Methods Comprehensive Rural Health Services Project (CRHSP) located in northern India, caters to a population of 70000 spread in 28 villages. All the individuals in the project area have been assigned a 10 digit unique identification number. Every year a health census is carried out to update the computerised health management information system by multi-purpose health workers. Quality control measure of such activity amounts cross verification of records of $25 \%$ population by the medical officer. The whole process takes 2 months time. LOAS was applied as a quality control measure for census operation. Village was considered as a Lot. $25 \%$ individual IDs were selected randomly from each village for quality check of demographic and health records. Allowable error was fixed at $5 \%$. If the error exceeded the limit of $5 \%$ the Lot was considered to be rejected and the whole census process was repeated.

Results Four villages were rejected upon using LOAS. The total duration of census operation was similar to previous method.

Conclusion LOAS can be used a scientific and robust method of quality control of census verification.

\section{P1-46 DIAGNOSTIC RADIATION EXPOSURE AND BREAST CANCER RISK IN BRCA1/2 MUTATION CARRIERS IN THE GENE-RAD- RISK STUDY}

doi:10.1136/jech.2011.142976c.39

${ }^{1} \mathrm{~A}$ Pijpe, ${ }^{*}{ }^{2} \mathrm{~N}$ Andrieu, ${ }^{3} \mathrm{D}$ Easton, ${ }^{4} \mathrm{~A}$ Kesminiene, ${ }^{5} \mathrm{E}$ Cardis, ${ }^{6} \mathrm{Genepso}$ study group, ${ }^{6} \mathrm{C}$ Nogues, ${ }^{3}$ Embrace study group, ${ }^{3} \mathrm{~S}$ Peock, ${ }^{3} \mathrm{D}$ Frost, ${ }^{1}$ Hebon study group, ${ }^{7} \mathrm{P}$ Manders, ${ }^{4} \mathrm{I}$ Thierry-Chef, ${ }^{8} \mathrm{D}$ Goldgar, ${ }^{1} \mathrm{M}$ Hauptmann, ${ }^{1} \mathrm{M}$ Rookus, ${ }^{1} \mathrm{~F}$ van Leeuwen. ${ }^{1}$ Netherlands Cancer Institute, Amsterdam, The Netherlands; ${ }^{2}$ Institut Curie, Paris, France; ${ }^{3}$ University of Cambridge, Cambridge, UK; ${ }^{4}$ IARC, Lyon, France; ${ }^{5}$ CREAL, Barcelona, Spain; ${ }^{6}$ Centre René Huguenin, Saint-Cloud, France; ${ }^{7}$ Radboud University Nijmegen Medical Centre, Nijmegen, The Netherlands; ${ }^{8}$ University of Utah, School of Medicine, Salt Lake City, USA

Introduction BRCA1/2 mutation carriers might be more sensitive to ionising radiation due to impaired DNA repair mechanisms.

Methods A retrospective European collaborative cohort study (GENE-RAD-RISK) of 1993 female BRCA1/2 mutation carriers was performed using self-reported exposure to diagnostic radiation. Risk of breast cancer was estimated using a weighted Cox proportional hazards model with cumulative radiation exposure from diagnostic procedures as a time-dependent variable lagged by 5 years.

Results are not presented in the abstract due to a publication embargo. 